\title{
新型手性三联吡啶钉(II)配合物催化酮到醇的氢转移反应
}

$\begin{array}{ccccc}\text { 王小蝶 } & \begin{array}{l}\text { 刘春玉 } \\ \text { 宋 苗玲玲 }\end{array} & \begin{array}{c}\text { 薛冰洁 } \\ \text { (郝新奇* }\end{array} & \text { 朱新学化学学院 } \\ & \text { 生命科学学院 } & \\ \text { 郑州 450001) } & \end{array}$

\author{
摘要 合成了一个含有手性对称三联吡啶配体的新型阳离子钓配合物，并通过核磁共振、高分辨质谱和 X 射线单晶衍 \\ 射确证了其结构. 该配合物在酮的氢转移中表现出较好的催化活性. 在最优条件下, 各种酮都可以在相对温和的条件 \\ 下顺利通过氢化反应高效地生成相应的醇，最高收率为 $99 \%$.
}

关键词 钓(II)配合物; 手性三联吡啶; 氢转移

\section{Transfer Hydrogenation of Ketones into Alcohols Catalyzed by a Novel Chiral Terpyridine Ruthenium(II) Complex}

\author{
Wang, Xiaodie Liu, Chunyu Miao, Lingling Xue, Bingjie \\ Zhu, Xinju Song, Bing* Hao, Xinqi* Liu, Guoji \\ (School of Life Sciences, College of Chemistry, Zhengzhou University, Zhengzhou 450001)
}

\begin{abstract}
A novel cationic ruthenium(II) complex bearing a chiral symmetrical pineno-fused terpyridine ligand was synthesized and structurally characterized by NMR, HRMS, and X-ray crystallographic determinations. This complex exhibited good catalytic activity in the transfer hydrogenation of ketones. Under optimized conditions, a wide range of ketones underwent hydrogenation smoothly to furnish corresponding alcohols in excellent yields (up to 99\%) under relative mild reaction conditions.

Keywords $\mathrm{Ru}(\mathrm{II})$ complexe; chiral terpyridine; transfer hydrogenation
\end{abstract}

\section{Introduction}

Reduction of carbonyl functionality to alcohols has become one of the most practical and fundamental transformations in synthetic organic chemistry. ${ }^{[1]}$ Among various existing methodology, transfer hydrogenation has been proved to be an efficient, mild and reliable method, which employs green and commercially available alcohols as the hydrogen donor. ${ }^{[2]}$ Noyori group ${ }^{[3]}$ discovered that $\mathrm{Ru}(\mathrm{II})$ complexes bearing $N$-tosyldiphenylethylene ligands exhibited high efficiency for transfer hydrogenation of ketones. Afterwards, Baratta group ${ }^{[4]}$ reported 2-(aminomethyl)pyridine-containing $\mathrm{Ru}(\mathrm{II})$ and $\mathrm{Os}(\mathrm{II})$ complexes, which also showed good catalytic activity. Meanwhile, Morris groups $^{[5]}$ developed tetradentate NNPP Fe(II) complexes, which initiated the utilization of earth-abundant transition metals in this field. In most cases, a rational ligand design is prerequisite in order to obtain transition metal complex- es with superior activity and selectivity. It is thus highly desirable to explore new types of ligands and the corresponding metal complexes to enhance the reaction efficiency.

Pincer-type complexes are a class of well-defined structures with superior performance in catalytic (de)hydrogenation and transfer (de)hydrogenation via fine modifications of steric and electronic effects. ${ }^{[6]} \mathrm{Up}$ to now, a series of NCN, ${ }^{[7]} \mathrm{PCP},{ }^{[8]} \mathrm{NNC},{ }^{[4,9]} \mathrm{PNP},{ }^{[10]} \mathrm{PNN},{ }^{[11]}$ and other ${ }^{[12]}$ tridentate metal complexes have been successfully utilized in transfer hydrogenation of ketones through vibration of donor moieties. In special, pincer complexes supported by NNN ligands, including bis(oxazolinyl)pyridines, ${ }^{[13]}$ bis(imidazolinyl)pyridines, ${ }^{[14]}$ and terpyridines, ${ }^{[15]}$ have exhibited superior reactivity. Yu and co-workers ${ }^{[16]}$ have developed various NNN pincer complexes and evaluated their performance in transfer hydrogenation of ketones

* Corresponding authors. E-mail: bingsong@zzu.edu.cn; xqhao@zzu.edu.cn

Received December 4, 2020; revised December 7, 2020; published online December 8, 2020.

Project supported by the National Natural Science Foundation of China (Nos. 21672192, 21803059, U1904212).

国家自然科学基金(Nos. 21672192, 21803059, U1904212)资助项目. 
with TOF values up to $1.4 \times 10^{7} \mathrm{~h}^{-1}$.

Over the past years, our group have developed a series of pincer complexes bearing imidazoline and imidazopyridine donors, which exhibited good catalytic activities in transfer hydrogenations of acetones, $\mathrm{N}$-alkylation of amines, $\alpha$-alkylation of acetone and nitriles, and other applications. ${ }^{[17-18]}$ As a continuation of our work, we herein report synthesis of a novel NNN cationic Ru(II) complex bearing a chiral symmetrical pineno-fused terpyridine ligand (Figure 1). Moreover, Ru(II)-catalyzed transfer hydrogenation of ketones is also presented.

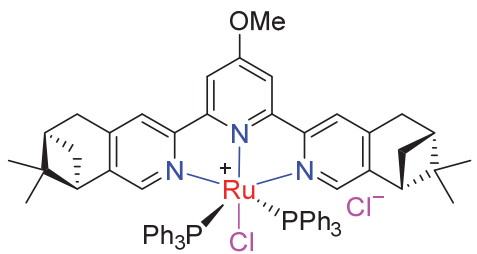

Chiral terpyridine $\mathrm{Ru}(\mathrm{II})$ complex 9

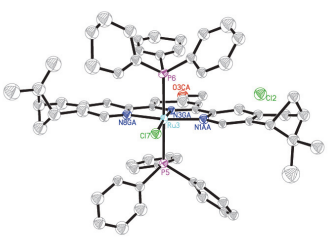

ORTEP of Ru(II) complex

Figure 1 Chiral terpyridine $\mathrm{Ru}(\mathrm{II})$ complex and its X-ray structure

\section{Results and discussion}

\subsection{Preparation of ligand and Ru complex}

The synthetic route of chiral terpyridine ligand $\mathbf{8}$ is illustrated in Scheme 1. Claisen condensation between commercially available diethyl oxalate $\mathbf{1}$ and acetone $\mathbf{2}$, followed by keto-enol tautomerism and dehydration generated ring-closing product 3 in $60 \%$ yield. After treatment with aqueous ammonia, 4-hydroxypyridine-2,6-dicarboxylic acid 4 was obtained in $93 \%$ yield. Next, esterification of 4 with alcohol would provide dimethyl 4-methoxypyridine-2,6-dicarboxylate $\mathbf{5}$, which reacted with ethyl acetate and metallic sodium followed by addition of $20 \%$ aqueous $\mathrm{HCl}$ to deliver 4-methoxy-2,6-diacetylpyridine 6 in $47 \%$ yield. After treatment with anhydrous pyridine and iodine at $100{ }^{\circ} \mathrm{C}$ for $3 \mathrm{~h}$, the corresponding product 7 was isolated in $76 \%$ yield. Finally, a Kröhnke-type reaction of $N$-heteropyridinium salt 7 with $(R)-(-)$-myrtenal in the presence of $\mathrm{NH}_{4} \mathrm{OAc}$ would gave chiral terpyridine ligand 8 in $70 \%$ yield, which underwent enolization of $N$-heteropyridinium salt 7, Michael addition of $(R)-(-)$-myrtenal with enolized 7, removal of pyridine group, and nucleophilic addition of $\mathrm{NH}_{4} \mathrm{OAc}$ process. ${ }^{[19]}$

With ligand $\mathbf{8}$ in hand, the desired pincer complex 9 was obtained in $68 \%$ yield after reacted with $\mathrm{RuCl}_{2}\left(\mathrm{PPh}_{3}\right)_{3}$ in $i$-PrOH under Ar for $3 \mathrm{~h}$ (Scheme 2). The structure of Ru(II) complex 9 was unambiguously determined by in-

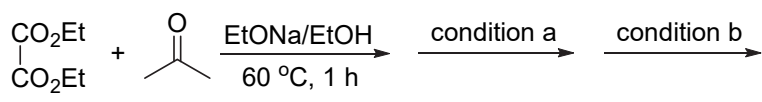

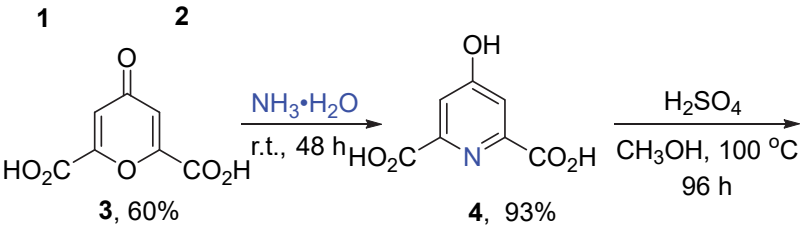



5, $29 \%$
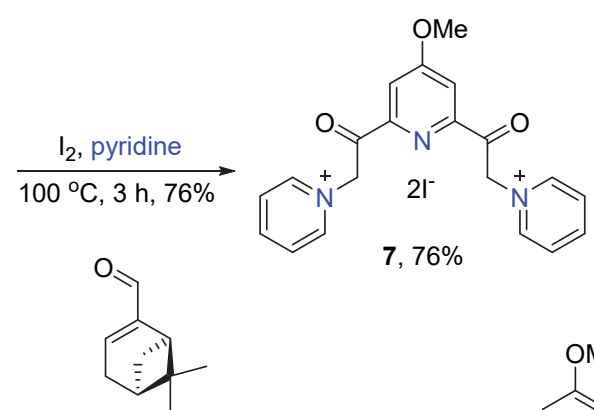

$R$-(-)-Myrtenal, $\mathrm{NH}_{4} \mathrm{OAc}$ $\mathrm{HCONH}_{2}, \mathrm{Ar}, 80^{\circ} \mathrm{C}, 12 \mathrm{~h}$

Condition a: $\mathrm{HCl}, \mathrm{H}_{2} \mathrm{O}, 50^{\circ} \mathrm{C}, 24 \mathrm{~h}$

Condition b: $\mathrm{HCl}, \mathrm{H}_{2} \mathrm{O}, 50^{\circ} \mathrm{C}, 72 \mathrm{~h}$

Scheme 1 Synthesis of chiral terpyridine ligand 8

frared spectrum, UV-Vis spectrum, NMR spectra and single-crystal X-ray diffraction analysis.
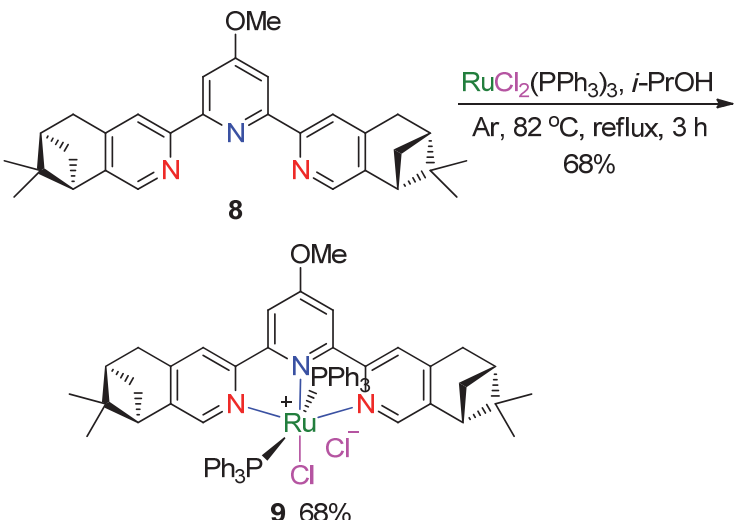

Scheme 2 Synthesis of $\mathrm{Ru}(\mathrm{II})$ complex 9

\subsection{Characterization of Ru complex}

${ }^{1} \mathrm{H}$ NMR analysis of complex 9 is consistent with molecular composition. After completion, the terpyridine proton signal of ligand 8 at $\delta 8.39(2 \mathrm{H})$ shifted downfield $(\delta 8.55)$ due to the decreased electron density upon coordination with $\mathrm{Ru}$ metal. On the contrary, proton signals of 8 at $\delta 8.21(2 \mathrm{H})$ and $7.94(2 \mathrm{H})$ shifted upfield $(\delta 8.09$ and $7.36)$, which could be explained by electron shielding ef- 
fect. ${ }^{[20]}$ Compared with ligand $\mathbf{8}$, the appearance of proton signal at $\delta 7.25 \sim 7.15(18 \mathrm{H})$ and $7.10 \sim 7.00(12 \mathrm{H})$ corresponded to $\mathrm{PPh}_{3}$ moiety in complex 9. In additional, ${ }^{31} \mathrm{P}$ NMR signal of complex 9 was also observed at $\delta$ 21.2. For both ligand 8 and complex 9 , proton signals at $\delta 4.01(3 \mathrm{H})$ and $4.20(3 \mathrm{H})$ were observed, respectively, which were assigned to methoxy group. Meanwhile, proton signals at $\delta$ $1.43(6 \mathrm{H})$ and $0.68(6 \mathrm{H})$ were corresponding to methyl group ligand 8. After complexion, the corresponding methyl proton signals located at $\delta 1.36(6 \mathrm{H})$ and $0.27(6 \mathrm{H})$ for complex 9. Finally, the remaining proton $(12 \mathrm{H})$ at the range of $\delta 0.79 \sim 3.12$ was also observed.

\subsection{X-ray crystallographic studies}

The molecular structure of complex 9 was further confirmed by single-crystal X-ray analysis (Figure 2). In the solid state, the six-coordinated cationic $\mathrm{Ru}(\mathrm{II})$ center is surrounded by the ligand $\mathbf{8}$, two $\mathrm{PPh}_{3}$ groups and one chloride atom in a distorted pseudo-octahedral configuration. The N(1AA)-Ru(3)-N(3GA) (78.2 $\left.{ }^{\circ}\right), \quad \mathrm{N}(3 \mathrm{GA})-\mathrm{Ru}(3)-$ $\mathrm{N}(8 \mathrm{GA})\left(78.4^{\circ}\right)$, and $\mathrm{N}(1 \mathrm{AA})-\mathrm{Ru}(3)-\mathrm{N}(8 \mathrm{GA})\left(156.6^{\circ}\right)$ angels deviate from the perfect values $\left(90^{\circ}\right.$ and $\left.180^{\circ}\right)$, indicating a constrained polycyclic ring system in the equatorial plane (Table 1). In contrast, the $\mathrm{P}(6)-\mathrm{Ru}(3)-\mathrm{N}(3 \mathrm{GA})$ $\left(90.1^{\circ}\right), \mathrm{N}(3 \mathrm{GA})-\mathrm{Ru}(3)-\mathrm{Cl}(7)\left(176.3^{\circ}\right)$, and $\mathrm{P}(5)-\mathrm{Ru}(3)-$ $\mathrm{P}(6)\left(179.1^{\circ}\right)$ angles are very approximate to $90^{\circ}$ and $180^{\circ}$, which suggest $\mathrm{Cl}(1)$ is positioned trans to the $\mathrm{N}(1)$ atom and two phosphorus atoms occupy the vertical coordination site.

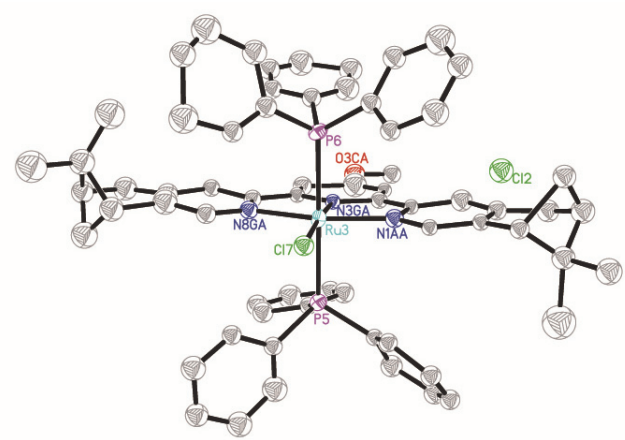

Figure 2 ORTEP drawing of chiral terpyridine ruthenium(II) complex

Table 1 Selected bond lengths and angles for complex 9

\begin{tabular}{lclc}
\hline \multicolumn{1}{c}{ Atom-Atom } & Length/nm & \multicolumn{1}{c}{ Atom-Atom-Atom } & Angle/( $\left.{ }^{\circ}\right)$ \\
\hline $\mathrm{Cl}(7)-\mathrm{Ru}(3)$ & 0.2458 & $\mathrm{~N}(3 \mathrm{GA})-\mathrm{Ru}(3)-\mathrm{Cl}(7)$ & 176.3 \\
$\mathrm{~N}(1 \mathrm{AA})-\mathrm{Ru}(3)$ & 0.2074 & $\mathrm{~N}(1 \mathrm{AA})-\mathrm{Ru}(3)-\mathrm{N}(8 \mathrm{GA})$ & 156.6 \\
$\mathrm{~N}(3 \mathrm{GA})-\mathrm{Ru}(3)$ & 0.1937 & $\mathrm{P}(5)-\mathrm{Ru}(3)-\mathrm{P}(6)$ & 179.1 \\
$\mathrm{~N}(8 \mathrm{GA})-\mathrm{Ru}(3)$ & 0.2129 & $\mathrm{P}(6)-\mathrm{Ru}(3)-\mathrm{N}(3 \mathrm{GA})$ & 90.1 \\
$\mathrm{P}(5)-\mathrm{Ru}(3)$ & 0.2407 & $\mathrm{~N}(1 \mathrm{AA})-\mathrm{Ru}(3)-\mathrm{N}(3 \mathrm{GA})$ & 78.2 \\
$\mathrm{P}(6)-\mathrm{Ru}(3)$ & 0.2412 & $\mathrm{~N}(3 \mathrm{AA})-\mathrm{Ru}(3)-\mathrm{N}(8 \mathrm{GA})$ & 78.4 \\
\hline
\end{tabular}

\subsection{Catalytic transfer hydrogenation of ketones}

Our initial investigation started with employment of ketone 10a and $i$-PrOH as the model reductant in the presence of $\mathrm{Ru}$ (II) complex 9 (Table 2). To our delight, the desired product 11a was isolated in $23 \%$ yield in the presence of $\mathrm{Na}_{2} \mathrm{CO}_{3}(10 \mathrm{~mol} \%)$ in isopropanol at $82{ }^{\circ} \mathrm{C}$ for 3 h (Table 2, Entry 1). Subsequently, various bases, including $\mathrm{KHCO}_{3}, \mathrm{~K}_{3} \mathrm{PO}_{4}, \mathrm{NaOAc}, \mathrm{NaOH}$ and EtONa, were tested, which indicated EtONa displayed the best activity to afford 11a in $87 \%$ yield (Table 2, Entries 2 6). Variation of base loading led to 11a in a decreased yield (Table 2, Entries 7 9). Next, the Ru(II) complex loading and reaction time was also modulated, which all suppressed reaction efficiency (Table 2, Entries 10 15). Finally, either elevating or lowering temperature was detrimental to the reaction.

Table 2 Optimization of reaction conditions ${ }^{a}$

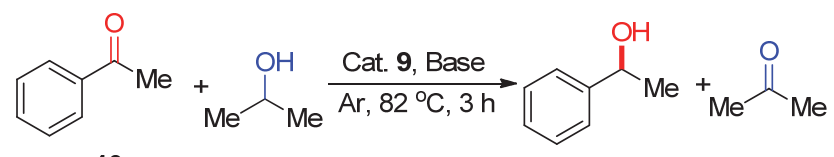

$10 a$

$11 a$

\begin{tabular}{ccccc}
\hline Entry & Base (equiv.) & Cat. 9/equiv. & Time/h & Yield/\% \\
\hline 1 & $\mathrm{Na}_{2} \mathrm{CO}_{3}(0.1)$ & 0.01 & 3 & 23 \\
2 & $\mathrm{KHCO}_{3}(0.1)$ & 0.01 & 3 & 47 \\
3 & $\mathrm{~K}_{3} \mathrm{PO}_{4}(0.1)$ & 0.01 & 3 & 74 \\
4 & $\mathrm{NaOAc}(0.1)$ & 0.01 & 3 & $\mathrm{~N} . \mathrm{R}$ \\
5 & $\mathrm{NaOH}(0.1)$ & 0.01 & 3 & 79 \\
$\mathbf{6}$ & EtONa (0.1) & $\mathbf{0 . 0 1}$ & $\mathbf{3}$ & $\mathbf{8 7}$ \\
7 & EtONa (0) & 0.01 & 3 & $\mathrm{~N} . \mathrm{R}$ \\
8 & EtONa (0.05) & 0.01 & 3 & 81 \\
9 & EtONa (0.2) & 0.01 & 3 & 78 \\
10 & EtONa (0.1) & 0.003 & 3 & 62 \\
11 & EtONa (0.1) & 0.005 & 3 & 82 \\
12 & EtONa (0.1) & 0.03 & 3 & 79 \\
13 & EtONa (0.1) & 0.05 & 3 & 66 \\
14 & EtONa (0.1) & 0.01 & 2 & 82 \\
15 & EtONa (0.1) & 0.01 & 4 & 65 \\
\hline
\end{tabular}

${ }^{a}$ Reaction conditions: ketone $(0.2 \mathrm{mmol}, 0.1 \mathrm{~mol} / \mathrm{L}$ in $2 \mathrm{~mL}$ of $i-\mathrm{PrOH})$; $n$ (ketone) $/ n$ (base) $/ n$ (cat. 9) $=100 / 10 / 1$, Ar $(0.1 \mathrm{MPa}), 82{ }^{\circ} \mathrm{C}, 3 \mathrm{~h}$, isolated yield.

With the optimized conditions in hand (Table 2, Entry 6 ), the substrate scope of ketones was investigated (Table $3)$. In general, various ortho-, meta-, and para-substituted aromatic methyl ketones could undergo transfer hydrogenation smoothly to give alcohols $11 \mathbf{a} \sim 11 \mathbf{1}$ in up to $99 \%$ yield. Both electron-donating (OMe, Me, Et and $t$-Bu) and electron-withdrawing $\left(\mathrm{CF}_{3}, \mathrm{Cl}\right.$ and $\left.\mathrm{Br}\right)$ groups were well-tolerated under the current catalytic system. However, a substantial decreased yield was observed when $\mathrm{NH}_{2}$ and $\mathrm{NO}_{2}$ substituents were introduced. Meanwhile, other ketones $10 \mathbf{r} \sim 10 \mathrm{t}$ were also tested to afford the corresponding alcohols in 95\% 99\% yields. The enantiomeric excess of product $\mathbf{1 0}$ was further determined by chiral high-performance liquid chromatography. Unfortunately, no enantioselectivity was observed probably due to the fact that the chiral environment of $\mathrm{Ru}$ complex 9 was far away from the substrates in the transfer hydrogenation process. 
Table 3 Substrate scope of ketones ${ }^{a}$

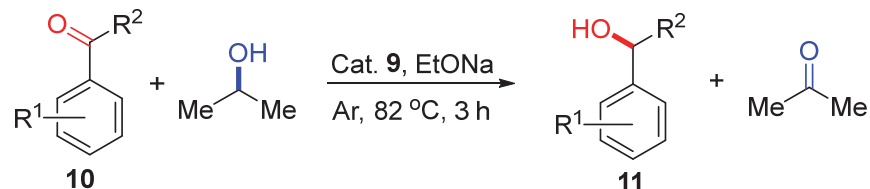<smiles>COc1ccccc1C(C)O</smiles>

11f, $25 \%$
11 e, $95 \%$<smiles>CC(O)c1ccccc1C(F)(F)F</smiles>

$11 \mathrm{~g}, 96 \%$ 11a, $87 \%$<smiles>COc1cccc(C(C)O)c1</smiles>

11h, $97 \%$

11b, $91 \%$<smiles>Cc1ccccc1C(O)O</smiles>

11d, $94 \%$<smiles>CC(O)c1cccc([N+](=O)[O-])c1</smiles>

$11 i, 12 \%$<smiles>CC(O)c1cccc(Cl)c1</smiles>

11j, $99 \%$<smiles>CC(O)c1cccc(Br)c1</smiles>

$11 k, 99 \%$<smiles>O=[N+]([O-])c1ccc(Cl)cc1</smiles>

11I, $96 \%$<smiles>CCc1ccc(C(C)O)cc1</smiles>

$11 \mathrm{~m}, 94 \%$<smiles>COc1ccc(C(C)O)cc1</smiles>

$\mathrm{Bu}$

11 n, $97 \%$<smiles>CC(O)c1ccc([N+](=O)[O-])cc1</smiles>

$11 q, 16 \%$<smiles>CCC(O)c1ccccc1</smiles>

$11 r, 95 \%$<smiles>CC(O)c1ccc2ccccc2c1</smiles>

11s, $99 \%$<smiles>OC(c1ccccc1)c1ccccc1</smiles>

$11 \mathrm{t}, 98 \%$

${ }^{a}$ Reaction conditions: ketone $(0.2 \mathrm{mmol}, 0.1 \mathrm{~mol} / \mathrm{L}$ in $2 \mathrm{~mL}$ of $i$-PrOH $) ; n$ (ketone) $/ n$ (base) $/ n$ (cat. 9) $=100 / 10 / 1, \mathrm{Ar}(0.1 \mathrm{MPa}), 82{ }^{\circ} \mathrm{C}, 3 \mathrm{~h}$, isolated yield.

To explore the reaction mechanism, an external amount of $\mathrm{PPh}_{3}$ was added to the reaction system (Scheme 3). The reaction efficiency was substantially suppressed in the presence of $1 \mathrm{~mol} \% \mathrm{PPh}_{3}$, which gave product 11a in 34\% yield. Further addition of $\mathrm{PPh}_{3}$ fully suppressed transfer hydrogenation of ketone 10a, indicating that dissociation of $\mathrm{PPh}_{3}$ ligand from Ru center is not involved. ${ }^{[18 \mathrm{a}]}$

On the basis of previous literature, ${ }^{[16,18]}$ a plausible mechanism was proposed (Scheme 4). Initially, Ru comp-

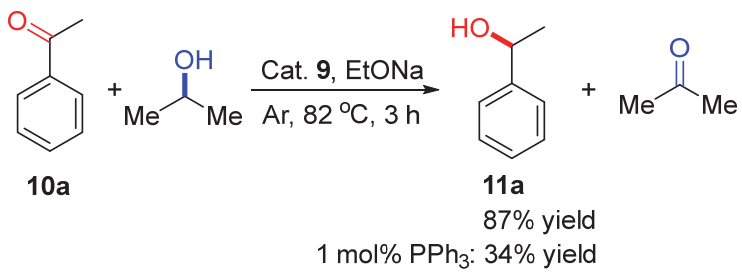

$5 \mathrm{~mol} \%, 10 \mathrm{~mol} \%$, or $20 \mathrm{~mol} \% \mathrm{PPh}_{3}$ : $0 \%$ yield

Scheme 3 Control experiments

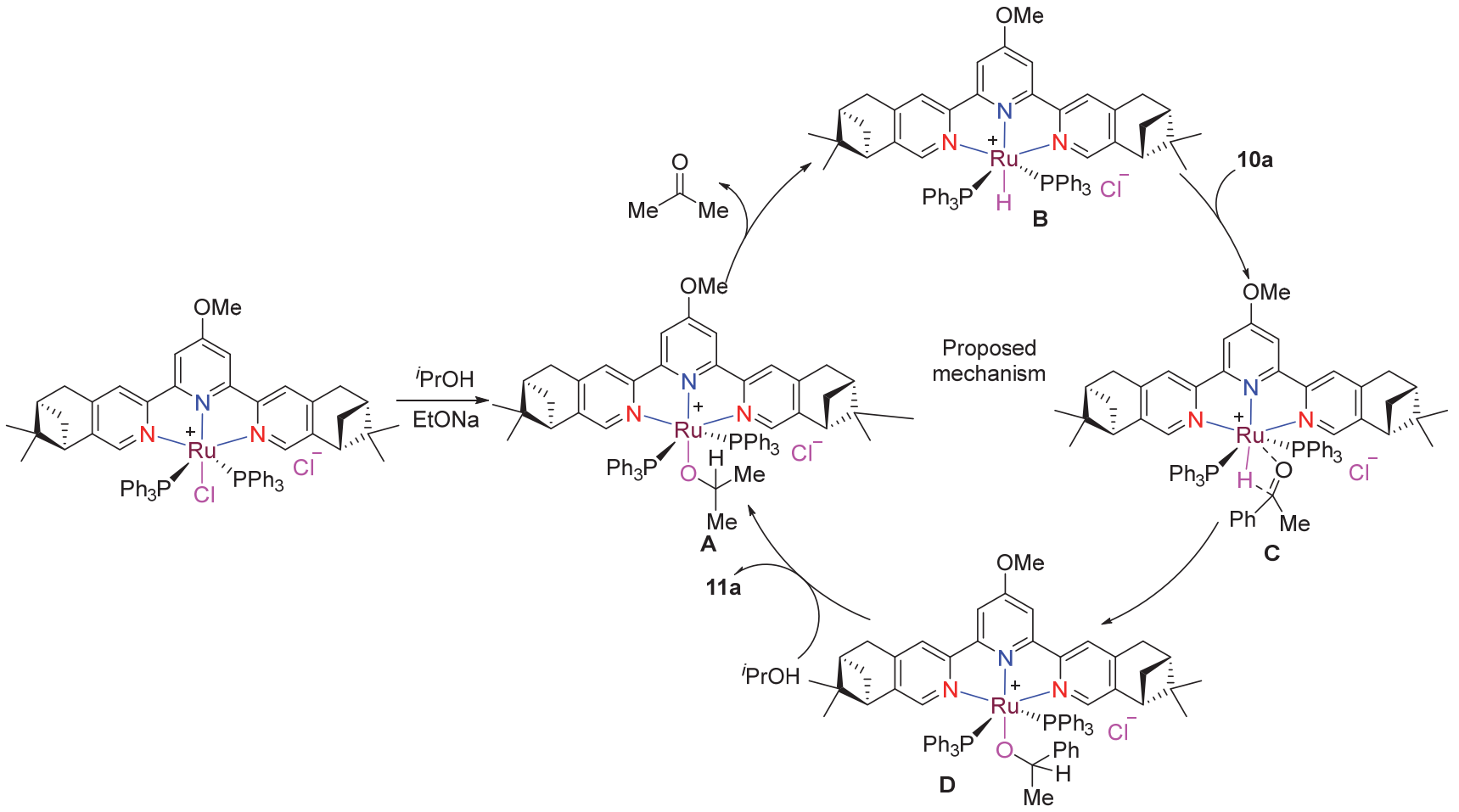

Scheme 4 Reaction mechanism 
lex 9 reacted with isopropyl alcohol in the presence of EtONa gave $\mathrm{Ru}(\mathrm{II})$ alkoxide intermediate $\mathbf{A}$, which underwent $\beta$-elimination to form $\mathrm{Ru}-\mathrm{H}$ intermediate $\mathbf{B}$. Coordination and insertion of ketone $\mathbf{1 0 a}$ to the $\mathrm{Ru}-\mathrm{H}$ bond provided $\mathrm{Ru}(\mathrm{II})$ alkoxide intermediate $\mathbf{C}$, which underwent alcohol metathesis to provide the desired product 11a and regenerate $\mathrm{Ru}(\mathrm{II})$ intermediate $\mathbf{A}$.

\section{Conclusions}

In conclusion, a novel chiral terpyridine ruthenium(II) complex, which was bench-stable and fully characterized, was successfully developed. The obtained $\mathrm{Ru}(\mathrm{II})$ catalyst exhibited good reactivity in transfer hydrogenation of ketones characterized with broad substrate scope, operational convenience, and high efficiency.

\section{Experimental section}

\subsection{General Information}

Melting points were determined on an XT4A melting point apparatus and were uncorrected. Flash column chromatography was performed using $200 \sim 300$ mesh silica gel. Analytical and preparative thin-layer chromatography (TLC) plates coated with commercial silica gel GF254 were used to monitor the reactions and purify products. ${ }^{1} \mathrm{H}$ NMR, ${ }^{13} \mathrm{C}$ NMR and ${ }^{19} \mathrm{~F}$ NMR, ${ }^{31} \mathrm{P}$ NMR spectra were recorded on Bruker DPX 400 or Bruker DPX 600 instruments using TMS as an internal standard. HRMS were determined on a Q-Tof Micro MS/MS System ESI spectrometer. HPLC analysis was performed on an Agilent Technologies instrument, employing a Daicel Chiralpak column at $25{ }^{\circ} \mathrm{C}$. The structure of Ru complex 9 (CCDC 1976681) was further confirmed by X-ray diffraction collected on a diffractometer with graphite monochromated $\mathrm{Mo} \mathrm{K} \alpha$ radiation. Unless otherwise indicated, all the starting materials and reagents were commercially available and used without further purification. Transfer hydrogenation of ketones was carried out in oven-dried $15 \mathrm{~mL}$ sealed tubes under Ar.

\subsection{General procedure for synthetic procedures}

Compounds $4 \sim 6$ were prepared according to Ref. [22] and the characterization data were consistent with the literature.

\subsubsection{Synthesis of compound 7}

To a $50 \mathrm{~mL}$ round-bottom flask was added compound $\mathbf{6}$ $(1.16 \mathrm{~g}, 6.0 \mathrm{mmol})$ and sublimated iodine $(3.12 \mathrm{~g}, 12.2$ $\mathrm{mmol})$ in dry pyridine $(9 \mathrm{~mL})$. The reaction mixture was stirred at $100{ }^{\circ} \mathrm{C}$ for $3 \mathrm{~h}$. After cooling down to room temperature, the precipitate was filtered, rinsed once with pyridine and dried under vacuo. Compound 7 was purified by recrystallization using $95 \%$ ethanol as a brown solid. 2.75 g, 76\% yield. m.p. $198 \sim 199{ }^{\circ} \mathrm{C} ;{ }^{1} \mathrm{H}$ NMR $(400$ MHz, DMSO- $\left.d_{6}\right) \delta: 9.10(\mathrm{~d}, J=5.2 \mathrm{~Hz}, 4 \mathrm{H}), 8.79(\mathrm{t}, J=$ $7.8 \mathrm{~Hz}, 2 \mathrm{H}), 8.34$ (t, $J=7.1 \mathrm{~Hz}, 4 \mathrm{H}), 7.89$ (s, 2H), 6.63 (s, $4 \mathrm{H}), 4.06(\mathrm{~s}, 3 \mathrm{H}) ;{ }^{13} \mathrm{C}$ NMR $\left(151 \mathrm{MHz}, \mathrm{CDCl}_{3}\right) \delta: 190.7$, $168.4,152.3,147.2,146.8,128.4,112.5,67.1,57.5,56.5$,
19.0; ${ }^{13} \mathrm{C}$ NMR $\left(151 \mathrm{MHz}, \mathrm{CDCl}_{3}\right) \delta: 190.7,168.4,152.3$, 147.2, 146.8, 128.4, 112.5, 67.1, 57.5, 56.5, 19.0; HRMS (ESI) calcd for $\mathrm{C}_{30} \mathrm{H}_{33} \mathrm{~N}_{3} \mathrm{O}[\mathrm{M}-\mathrm{I}]^{+}$476.0466, found 476.0466 .

\subsubsection{Synthesis of compound $\mathbf{8}$}

To a $100 \mathrm{~mL}$ schlenk-bottle was added a solution of compound 7 (1.20 g, $1.99 \mathrm{mmol})$, ammonium acetate (0.66 $\mathrm{g}, 4.17 \mathrm{mmol})$ and $(R)$-myrtenal $(608 \mu \mathrm{L}, 4.03 \mathrm{mmol})$ in formamide $(6 \mathrm{~mL})$ under nitrogen. The reaction mixture was stirred at $80{ }^{\circ} \mathrm{C}$ overnight. After cooling down to room temperature, the precipitate was filtered, rinsed once with water, and dried under vacuo. The residue was purified by neutral alumina chromatography (petroleum ether/ ethyl acetate, $V: V=50: 1,1 \%$ triethylamine as the eluent) to give compound 8 as a light yellow solid. $0.63 \mathrm{~g}$, $70 \%$ yield. m.p. $231 \sim 232{ }^{\circ} \mathrm{C}$; ${ }^{1} \mathrm{H}$ NMR $(400 \mathrm{MHz}$, $\left.\mathrm{CDCl}_{3}\right) \delta: 8.39(\mathrm{~s}, 2 \mathrm{H}), 8.21(\mathrm{~s}, 2 \mathrm{H}), 7.94(\mathrm{~s}, 2 \mathrm{H}), 4.01(\mathrm{~s}$, $3 \mathrm{H}), 3.12(\mathrm{~d}, J=2.4 \mathrm{~Hz}, 4 \mathrm{H}), 2.88(\mathrm{t}, J=5.4 \mathrm{~Hz}, 2 \mathrm{H}), 2.72$ $(\mathrm{m}, J=9.7,5.8 \mathrm{~Hz}, 2 \mathrm{H}), 2.32 \sim 2.36(\mathrm{~m}, 2 \mathrm{H}), 1.43(\mathrm{~s}, 6 \mathrm{H})$, $1.26(\mathrm{~d}, J=9.6 \mathrm{~Hz}, 2 \mathrm{H}), 0.68(\mathrm{~s}, 6 \mathrm{H}) ;{ }^{13} \mathrm{C} \mathrm{NMR}(151 \mathrm{MHz}$, $\left.\mathrm{CDCl}_{3}\right) \delta: 167.7,157.6,154.5,145.4,145.2,143.0,120.7$, 106.4, 55.5, 44.6, 40.1, 39.3, 33.0, 31.9, 26.0, 21.4; HRMS (ESI) calcd for $\mathrm{C}_{30} \mathrm{H}_{33} \mathrm{~N}_{3} \mathrm{O}[\mathrm{M}+\mathrm{H}]^{+}$452.2696, found 452.2702 .

\subsubsection{Synthesis of pincer Ru(II) complex 9}

To a $25 \mathrm{~mL}$ Schlenk tube was added a solution of compound 8 (45 mg, $1.0 \mathrm{mmol}$ ) and $\mathrm{RuCl}_{2}\left(\mathrm{PPh}_{3}\right)_{3}$ (96 mg, 1.0 $\mathrm{mmol})$ in isopropanol $(10 \mathrm{~mL})$ under nitrogen. The reaction mixture was refluxed at $82{ }^{\circ} \mathrm{C}$ for $3 \mathrm{~h}$. After cooled down to room temperature, complex 9 was obtained via recrystallization followed by washing with anhydrous ether as a red solid. $78 \mathrm{mg}, 68 \%$ yield. m.p. $158 \sim 159{ }^{\circ} \mathrm{C} ;{ }^{1} \mathrm{H}$ NMR $\left(400 \mathrm{MHz}_{2} \mathrm{CDCl}_{3}\right) \delta: 8.55(\mathrm{~s}, 2 \mathrm{H}), 8.09(\mathrm{~s}, 2 \mathrm{H}), 7.36$ $(\mathrm{s}, 2 \mathrm{H}), 7.25 \sim 7.15(\mathrm{~m}, 18 \mathrm{H}), 7.05(\mathrm{t}, J=7.5 \mathrm{~Hz}, 12 \mathrm{H})$, $4.20(\mathrm{~s}, 3 \mathrm{H}), 3.10 \sim 2.99(\mathrm{~m}, 4 \mathrm{H}), 2.66 \sim 2.53(\mathrm{~m}, 4 \mathrm{H})$, $2.24 \sim 2.22(\mathrm{~m}, 2 \mathrm{H}), 1.36(\mathrm{~s}, 6 \mathrm{H}), 0.79(\mathrm{~d}, J=9.6 \mathrm{~Hz}, 2 \mathrm{H})$, $0.27(\mathrm{~s}, 6 \mathrm{H}) ;{ }^{13} \mathrm{C}$ NMR $\left(151 \mathrm{MHz}, \mathrm{CDCl}_{3}\right) \delta: 165.1,157.6$, $156.1,151.4,145.8,145.6,133.2$, 133.14, 133.10, 130.6, $130.5,130.4,129.4,127.9,127.88,127.85,122.9,109.4$, 59.1, 44.6, 39.5, 38.8, 32.7, 31.0, 25.8, 21.2; ${ }^{31} \mathrm{P}$ NMR $\left(162 \mathrm{MHz}, \mathrm{CDCl}_{3}\right) \delta: 21.16(\mathrm{~s})$; HRMS (ESI ${ }^{+}$) calcd for $\mathrm{C}_{66} \mathrm{H}_{62} \mathrm{ClN}_{3} \mathrm{OP}_{2} \mathrm{Ru}[\mathrm{M}+\mathrm{H}]{ }^{+} 1112.3173$, found 1112.3191 .

\subsubsection{Transfer hydrogenation of ketones}

The catalyst solution was prepared by dissolving $\mathrm{Ru}(\mathrm{II})$ complex 9 (11.5 mg, $0.01 \mathrm{mmol})$ in isopropanol $(10 \mathrm{~mL})$. To a $10 \mathrm{~mL}$ Schlenk tube was added compound $10(0.2$ $\mathrm{mmol})$, EtONa (1.4 mg, $0.02 \mathrm{mmol})$ in above catalyst solution $(2 \mathrm{~mL}, 0.002 \mathrm{mmol})$. The reaction mixture was heated at $82{ }^{\circ} \mathrm{C}$ for $3 \mathrm{~h}$ under $\mathrm{Ar}$, and then cooled down to $0{ }^{\circ} \mathrm{C}$. After removal of organic solvent, the desired product 11 were purified by preparative TLC on silica gel plates (petroleum ether/ethyl acetate, $V: V=10: 1$ as the eluent). ${ }^{[19 a]}$

$\alpha$-Methylbenzyl alcohol (11a): Slight yellow oil, 21.3 mg, 87\% yield. ${ }^{1} \mathrm{H}$ NMR (400 $\left.\mathrm{MHz}, \mathrm{CDCl}_{3}\right) \delta: 7.30$ (d, 
$J=4.3 \mathrm{~Hz}, 4 \mathrm{H}), 7.25 \sim 7.18(\mathrm{~m}, 1 \mathrm{H}), 4.79(\mathrm{q}, J=6.4 \mathrm{~Hz}$, $1 \mathrm{H}), 2.53(\mathrm{~s}, 1 \mathrm{H}), 1.42$ (d, $J=6.5 \mathrm{~Hz}, 3 \mathrm{H}) ;{ }^{13} \mathrm{C}$ NMR $(101$ $\left.\mathrm{MHz}, \mathrm{CDCl}_{3}\right) \delta: 145.9,128.5,127.4,125.5,70.3,25.2$.

2-Chloro- $\alpha$-methylbenzyl alcohol (11b): Slight yellow oil, $28.5 \mathrm{mg}$, 91\% yield. ${ }^{1} \mathrm{H}$ NMR (400 $\left.\mathrm{MHz}, \mathrm{CDCl}_{3}\right) \delta$ : $7.58(\mathrm{dd}, J=7.7,1.5 \mathrm{~Hz}, 1 \mathrm{H}), 7.34 \sim 7.24(\mathrm{~m}, 2 \mathrm{H}), 7.19$ (td, $J=7.6,1.7 \mathrm{~Hz}, 1 \mathrm{H}), 5.26(\mathrm{q}, J=6.2 \mathrm{~Hz}, 1 \mathrm{H}), 2.47$ (s, $1 \mathrm{H}), 1.47(\mathrm{~d}, J=6.4 \mathrm{~Hz}, 3 \mathrm{H}) ;{ }^{13} \mathrm{C} \mathrm{NMR}\left(101 \mathrm{MHz}, \mathrm{CDCl}_{3}\right)$ $\delta$ : 143.1, 131.6, 129.4, 128.4, 127.2, 126.4, 66.9, 23.5.

2-Bromo- $\alpha$-methylbenzyl alcohol (11c): Slight yellow oil, $34.6 \mathrm{mg}, 86 \%$ yield. ${ }^{1} \mathrm{H}$ NMR $\left(400 \mathrm{MHz}, \mathrm{CDCl}_{3}\right) \delta$ : 7.57 (dd, $J=7.8,1.7 \mathrm{~Hz}, 1 \mathrm{H}), 7.50$ (dd, $J=8.0,0.9 \mathrm{~Hz}$, $1 \mathrm{H}), 7.33(\mathrm{t}, J=7.7 \mathrm{~Hz}, 1 \mathrm{H}), 7.11(\mathrm{td}, J=7.7,1.7 \mathrm{~Hz}, 1 \mathrm{H})$, $5.21(\mathrm{q}, J=6.4 \mathrm{~Hz}, 1 \mathrm{H}), 2.48(\mathrm{~s}, 1 \mathrm{H}), 1.46(\mathrm{~d}, J=6.4 \mathrm{~Hz}$, $3 \mathrm{H}) ;{ }^{13} \mathrm{C}$ NMR (101 MHz, $\left.\mathrm{CDCl}_{3}\right) \delta: 144.6,132.6,128.7$, $127.8,126.7,121.7,69.2,23.60$.

1-(2-Methylphenyl)ethanol (11d): Slight yellow oil, 25.6 mg, 94\% yield. ${ }^{1} \mathrm{H}$ NMR (400 $\left.\mathrm{MHz}, \mathrm{CDCl}_{3}\right) \delta: 7.51 \sim 7.49$ $(\mathrm{m}, 1 \mathrm{H}), 7.27 \sim 7.13(\mathrm{~m}, 3 \mathrm{H}), 5.10(\mathrm{q}, J=6.4 \mathrm{~Hz}, 1 \mathrm{H})$, $2.34(\mathrm{~s}, 3 \mathrm{H}), 2.21(\mathrm{~s}, 1 \mathrm{H}), 1.46(\mathrm{~d}, J=6.4 \mathrm{~Hz}, 3 \mathrm{H}) ;{ }^{13} \mathrm{C}$ NMR $\left(101 \mathrm{MHz}, \mathrm{CDCl}_{3}\right) \delta: 143.9,134.2,130.4,127.1$, 126.4, 124.5, 66.7, 23.9, 18.9 .

2-Methoxy- $\alpha$-methylbenzyl alcohol (11e): Slight yellow oil, $28.9 \mathrm{mg}$, 95\% yield. ${ }^{1} \mathrm{H}$ NMR (400 $\left.\mathrm{MHz}, \mathrm{CDCl}_{3}\right) \delta$ : $7.36(\mathrm{dd}, J=7.5,1.6 \mathrm{~Hz}, 1 \mathrm{H}), 7.28 \sim 7.22(\mathrm{~m}, 1 \mathrm{H}), 6.98$ $(\mathrm{td}, J=7.5,0.9 \mathrm{~Hz}, 1 \mathrm{H}), 6.88(\mathrm{~d}, J=8.2 \mathrm{~Hz}, 1 \mathrm{H}), 5.10$ (q, $J=6.5 \mathrm{~Hz}, 1 \mathrm{H}), 3.86(\mathrm{~s}, 3 \mathrm{H}), 2.79$ (s, 1H), 1.51 (d, $J=6.5$ $\mathrm{Hz}, 3 \mathrm{H}) ;{ }^{13} \mathrm{C} \mathrm{NMR}\left(101 \mathrm{MHz}, \mathrm{CDCl}_{3}\right) \delta: 156.5,133.5$, $128.3,126.1,120.8,110.4,66.4,55.3,22.9$.

1-(2-Aminophenyl)ethanol (11f): Slight yellow oil, 6.9 mg, 25\% yield. ${ }^{1} \mathrm{H}$ NMR $\left(400 \mathrm{MHz}, \mathrm{CDCl}_{3}\right) \delta: 7.13 \sim 7.05$ $(\mathrm{m}, 2 \mathrm{H}), 6.76 \sim 6.64(\mathrm{~m}, 2 \mathrm{H}), 4.92(\mathrm{q}, J=6.6 \mathrm{~Hz}, 1 \mathrm{H})$, $1.59(\mathrm{~d}, J=6.6 \mathrm{~Hz}, 3 \mathrm{H}) ;{ }^{13} \mathrm{C} \mathrm{NMR}\left(101 \mathrm{MHz}, \mathrm{CDCl}_{3}\right) \delta$ : 145.1, 128.6, 128.3, 126.5, 118.1, 116.6, 69.6, 21.5.

$\alpha$-Methyl-2-(trifluoromethyl)benzyl alcohol (11g): Slight yellow oil, $36.9 \mathrm{mg}$, 96\% yield. ${ }^{1} \mathrm{H}$ NMR (400 MHz, $\left.\mathrm{CDCl}_{3}\right) \delta: 7.83(\mathrm{~d}, J=7.8 \mathrm{~Hz}, 1 \mathrm{H}), 7.64 \sim 7.55(\mathrm{~m}, 2 \mathrm{H})$, $7.37(\mathrm{t}, J=7.6 \mathrm{~Hz}, 1 \mathrm{H}), 5.35 \sim 5.31(\mathrm{~m}, 1 \mathrm{H}), 2.01(\mathrm{~s}, 1 \mathrm{H})$, $1.49(\mathrm{~d}, J=6.3 \mathrm{~Hz}, 3 \mathrm{H}) ;{ }^{13} \mathrm{C} \mathrm{NMR}\left(101 \mathrm{MHz}, \mathrm{CDCl}_{3}\right) \delta$ : 145.0, 132.4, $127.2\left(J_{\mathrm{C}-\mathrm{F}}=26.9 \mathrm{~Hz}\right), 126.5\left(J_{\mathrm{C}-\mathrm{F}}=30.2\right.$ $\mathrm{Hz}), 125.7,125.3\left(J_{\mathrm{C}-\mathrm{F}}=5.9 \mathrm{~Hz}\right), 123.0,65.7\left(J_{\mathrm{C}-\mathrm{F}}=2.4\right.$ $\mathrm{Hz}), 25.43 ;{ }^{19} \mathrm{~F} \mathrm{NMR}\left(376 \mathrm{MHz}, \mathrm{CDCl}_{3}\right) \delta$ : -58.32 .

1-(3-Methoxyphenyl)ethanol (11h): Slight yellow oil, $29.5 \mathrm{mg}, 97 \%$ yield. ${ }^{1} \mathrm{H}$ NMR $\left(400 \mathrm{MHz}, \mathrm{CDCl}_{3}\right) \delta: 7.29 \sim$ $7.22(\mathrm{~m}, 1 \mathrm{H}), 6.97 \sim 6.91(\mathrm{~m}, 2 \mathrm{H}), 6.81 \sim 6.79(\mathrm{~m}, 1 \mathrm{H})$, 4.86 (q, $J=6.4 \mathrm{~Hz}, 1 \mathrm{H}), 3.81$ (s, 3H), 1.99 (s, 1H), 1.49 (d, $J=6.5 \mathrm{~Hz}, 3 \mathrm{H}) ;{ }^{13} \mathrm{C} \mathrm{NMR}\left(101 \mathrm{MHz}, \mathrm{CDCl}_{3}\right) \delta: 159.8$, $147.6,129.5,117.7,112.9,110.9,70.3,55.2,25.2$.

1-(3-Nitrophenyl)ethanol (11i): Slight yellow oil, 4.0 mg, $12 \%$ yield. ${ }^{1} \mathrm{H}$ NMR (400 MHz, $\left.\mathrm{CDCl}_{3}\right) \delta: 8.26(\mathrm{t}, J=$ $1.9 \mathrm{~Hz}, 1 \mathrm{H}), 8.14 \sim 8.12(\mathrm{~m}, 1 \mathrm{H}), 7.72(\mathrm{~d}, J=7.7 \mathrm{~Hz}, 1 \mathrm{H})$, 7.53 (t, $J=7.9 \mathrm{~Hz}, 1 \mathrm{H}), 5.03$ (q, $J=6.4 \mathrm{~Hz}, 1 \mathrm{H}), 1.98$ (s, 1H), $1.55(\mathrm{~d}, J=6.5 \mathrm{~Hz}, 3 \mathrm{H}) ;{ }^{13} \mathrm{C} \mathrm{NMR}\left(101 \mathrm{MHz}, \mathrm{CDCl}_{3}\right)$ $\delta: 148.4,147.8,131.6,129.4,122.4,120.4,69.4,25.5$.

3-Chloro- $\alpha$-methylbenzyl alcohol (11j): Slight yellow oil, $31.0 \mathrm{mg}, 99 \%$ yield. ${ }^{1} \mathrm{H}$ NMR (400 $\left.\mathrm{MHz} \mathrm{CDCl}_{3}\right) \delta$ : $7.33(\mathrm{~s}, 1 \mathrm{H}), 7.25 \sim 7.17(\mathrm{~m}, 3 \mathrm{H}), 4.81(\mathrm{q}, J=6.4 \mathrm{~Hz}, 1 \mathrm{H})$, $2.31(\mathrm{~s}, 1 \mathrm{H}), 1.43(\mathrm{~d}, J=6.5 \mathrm{~Hz}, 3 \mathrm{H}) ;{ }^{13} \mathrm{C} \mathrm{NMR}(101 \mathrm{MHz}$, $\left.\mathrm{CDCl}_{3}\right) \delta: 147.9,134.3,129.8,127.5,125.6,123.5,69.7$, 25.2 .

3-Bromo- $\alpha$-methylbenzyl alcohol (11k): Slight yellow oil, $39.8 \mathrm{mg}, 99 \%$ yield. ${ }^{1} \mathrm{H}$ NMR (400 $\left.\mathrm{MHz} \mathrm{CDCl}_{3}\right) \delta$ : $7.50(\mathrm{~s}, 1 \mathrm{H}), 7.40 \sim 7.35(\mathrm{~m}, 1 \mathrm{H}), 7.28 \sim 7.23(\mathrm{~m}, 1 \mathrm{H})$, $7.19(\mathrm{t}, J=7.7 \mathrm{~Hz}, 1 \mathrm{H}), 4.81(\mathrm{q}, J=6.5 \mathrm{~Hz}, 1 \mathrm{H}), 2.31(\mathrm{~s}$, $1 \mathrm{H}), 1.44(\mathrm{~d}, J=6.5 \mathrm{~Hz}, 3 \mathrm{H}) ;{ }^{13} \mathrm{C} \mathrm{NMR}\left(101 \mathrm{MHz}, \mathrm{CDCl}_{3}\right)$ $\delta: 148.1,130.4,130.1,128.6,124.0,122.6,69.7,25.2$.

4-Chloro- $\alpha$-methylbenzyl alcohol (111): Slight yellow oil, $30.0 \mathrm{mg}$, 96\% yield. ${ }^{1} \mathrm{H}$ NMR (400 $\left.\mathrm{MHz}, \mathrm{CDCl}_{3}\right) \delta$ : $7.30 \sim 7.25(\mathrm{~m}, 4 \mathrm{H}), 4.83(\mathrm{q}, J=6.4 \mathrm{~Hz}, 1 \mathrm{H}), 2.33(\mathrm{~s}, 1 \mathrm{H})$, $1.44(\mathrm{~d}, J=6.5 \mathrm{~Hz}, 3 \mathrm{H}) ;{ }^{13} \mathrm{C} \mathrm{NMR}\left(101 \mathrm{MHz}, \mathrm{CDCl}_{3}\right) \delta$ : 144.3, 133.0, 128.6, 126.8, 69.7, 25.2.

1-(4-Ethylphenyl)ethanol (11m): Slight yellow oil, 28.2 mg, 94\% yield. ${ }^{1} \mathrm{H}$ NMR (400 $\left.\mathrm{MHz}, \mathrm{CDCl}_{3}\right) \delta: 7.31 \sim 7.29$ $(\mathrm{m}, 2 \mathrm{H}), 7.20(\mathrm{~d}, J=8.1 \mathrm{~Hz}, 2 \mathrm{H}), 4.87(\mathrm{q}, J=6.4 \mathrm{~Hz}, 1 \mathrm{H})$, 2.67 (q, $J=7.6 \mathrm{~Hz}, 2 \mathrm{H}), 2.07$ (s, 1H), 1.50 (d, $J=6.5 \mathrm{~Hz}$, $3 \mathrm{H}), 1.26(\mathrm{t}, J=7.6 \mathrm{~Hz}, 3 \mathrm{H}) ;{ }^{13} \mathrm{C} \mathrm{NMR}\left(101 \mathrm{MHz}, \mathrm{CDCl}_{3}\right)$ $\delta: 143.5,143.2,128.0,125.5,70.2,28.6,25.1,15.6$.

4-(tert-Butyl)- $\alpha$-methylbenzyl alcohol (11n): Slight yellow oil, $34.6 \mathrm{mg}, 97 \%$ yield. ${ }^{1} \mathrm{H}$ NMR (400 MHz, $\left.\mathrm{CDCl}_{3}\right) \delta: 7.41 \sim 7.31(\mathrm{~m}, 2 \mathrm{H}), 7.35 \sim 7.30(\mathrm{~m}, 2 \mathrm{H}), 4.87$ (q, $J=6.5 \mathrm{~Hz}, 1 \mathrm{H}), 2.10(\mathrm{~s}, 1 \mathrm{H}), 1.51(\mathrm{~d}, J=6.5 \mathrm{~Hz}, 3 \mathrm{H})$, $1.35(\mathrm{~s}, 9 \mathrm{H}) ;{ }^{13} \mathrm{C}$ NMR (101 MHz, $\left.\mathrm{CDCl}_{3}\right) \delta: 150.4,142.9$, $125.4,125.2,70.2,34.5,31.4,24.9$.

1-(4-Methoxyphenyl)ethanol (110): Slight yellow oil, $28.9 \mathrm{mg}, 95 \%$ yield. ${ }^{1} \mathrm{H} \mathrm{NMR}\left(400 \mathrm{MHz}, \mathrm{CDCl}_{3}\right) \delta: 7.30 \sim$ $7.25(\mathrm{~m}, 2 \mathrm{H}), 6.88 \sim 6.84(\mathrm{~m}, 2 \mathrm{H}), 4.81(\mathrm{q}, J=6.4 \mathrm{~Hz}$, $1 \mathrm{H}), 3.78$ (s, 3H), $2.14(\mathrm{~s}, 1 \mathrm{H}), 1.45$ (d, $J=6.5 \mathrm{~Hz}, 3 \mathrm{H})$; ${ }^{13} \mathrm{C}$ NMR $\left(101 \mathrm{MHz}, \mathrm{CDCl}_{3}\right) \delta: 158.9,138.1,126.7,113.8$, $69.9,55.3,25.0$.

1-(4-Methylphenyl)ethanol (11p): Slight yellow oil, 26.7 mg, 98\% yield. ${ }^{1} \mathrm{H}$ NMR (400 MHz, $\left.\mathrm{CDCl}_{3}\right) \delta: 7.25$ (d, $J=8.1 \mathrm{~Hz}, 2 \mathrm{H}), 7.15$ (d, $J=7.9 \mathrm{~Hz}, 2 \mathrm{H}), 4.84$ (q, $J=6.4$ $\mathrm{Hz}, 1 \mathrm{H}), 2.34$ (s, 3H), 1.92 (s, 1H), 1.46 (d, $J=6.5 \mathrm{~Hz}$, $3 \mathrm{H}) ;{ }^{13} \mathrm{C} \mathrm{NMR}\left(101 \mathrm{MHz}, \mathrm{CDCl}_{3}\right) \delta: 142.9,137.2,129.2$, $125.4,70.2,25.1,21.1$.

1-(4-Nitrophenyl)ethanol (11q): Slight yellow oil, 5.3 mg, $16 \%$ yield. ${ }^{1} \mathrm{H}$ NMR (400 $\left.\mathrm{MHz}, \mathrm{CDCl}_{3}\right) \delta: 8.25 \sim 8.15$ $(\mathrm{m}, 2 \mathrm{H}), 7.54$ (d, $J=8.5 \mathrm{~Hz}, 2 \mathrm{H}), 5.03$ (q, $J=6.5 \mathrm{~Hz}, 1 \mathrm{H})$, $1.98(\mathrm{~s}, 1 \mathrm{H}), 1.53(\mathrm{~d}, J=6.5 \mathrm{~Hz}, 3 \mathrm{H}) ;{ }^{13} \mathrm{C} \mathrm{NMR}(101 \mathrm{MHz}$, $\left.\mathrm{CDCl}_{3}\right) \delta: 153.0,147.2,126.1,123.8,69.5,25.5$.

1-Phenyl-1-propanol (11r): Slight yellow oil, $25.9 \mathrm{mg}$, 95\% yield. ${ }^{1} \mathrm{H}$ NMR $\left(400 \mathrm{MHz}, \mathrm{CDCl}_{3}\right) \delta: 7.37 \sim 7.30(\mathrm{~m}$, $4 \mathrm{H}), 7.30 \sim 7.23(\mathrm{~m}, 1 \mathrm{H}), 4.57(\mathrm{t}, J=6.6 \mathrm{~Hz}, 1 \mathrm{H}), 2.01(\mathrm{~s}$, $1 \mathrm{H}), 1.88 \sim 1.64(\mathrm{~m}, 2 \mathrm{H}), 0.91(\mathrm{t}, J=7.4 \mathrm{~Hz}, 3 \mathrm{H}) ;{ }^{13} \mathrm{C}$ NMR $\left(101 \mathrm{MHz}, \mathrm{CDCl}_{3}\right) \delta: 144.6,128.4,127.5,126.0$, $76.0,31.9,10.2$.

1-(2-Naphthyl)ethanol (11s): White solid, $34.1 \mathrm{mg}, 99 \%$ yield. m.p. $74 \sim 75{ }^{\circ} \mathrm{C}$; ${ }^{1} \mathrm{H}$ NMR $\left(400 \mathrm{MHz}, \mathrm{CDCl}_{3}\right) \delta$ : $7.85 \sim 7.80(\mathrm{~m}, 4 \mathrm{H}), 7.51 \sim 7.46(\mathrm{~m}, 3 \mathrm{H}), 5.06(\mathrm{q}, J=6.3$ $\mathrm{Hz}, 1 \mathrm{H}), 2.14(\mathrm{~s}, 1 \mathrm{H}), 1.58(\mathrm{~d}, J=6.5 \mathrm{~Hz}, 3 \mathrm{H}) ;{ }^{13} \mathrm{C} \mathrm{NMR}$ $\left(101 \mathrm{MHz}, \mathrm{CDCl}_{3}\right) \delta: 143.2,133.3,132.9,128.3,127.9$, $127.7,126.2,125.8,123.8,123.8,70.5,25.2$.

Benzhydrol (11t): White solid, $36.1 \mathrm{mg}, 98 \%$ yield. m.p. $65 \sim 66{ }^{\circ} \mathrm{C} ;{ }^{1} \mathrm{H}$ NMR $\left(400 \mathrm{MHz}, \mathrm{CDCl}_{3}\right) \delta: 7.39 \sim 7.27$ 
$(\mathrm{m}, 8 \mathrm{H}), 7.27 \sim 7.20(\mathrm{~m}, 2 \mathrm{H}), 5.78(\mathrm{~d}, J=3.0 \mathrm{~Hz}, 1 \mathrm{H})$, $2.35(\mathrm{~d}, J=3.4 \mathrm{~Hz}, 1 \mathrm{H}) ;{ }^{13} \mathrm{C} \mathrm{NMR}\left(101 \mathrm{MHz}, \mathrm{CDCl}_{3}\right) \delta$ : $143.8,128.5,127.6,126.6,76.3$.

Supporting Information ${ }^{1} \mathrm{H}$ NMR and ${ }^{13} \mathrm{C}$ NMR original spectra of the target compound. The Supporting Information is available free of charge via the Internet at http://sioc-journal.cn.

\section{References}

[1] (a) Trost, B. M.; Fleming, I. Comprehensive Organic Synthesis, Vol. 8, Pergamon, Oxford, U. K., 1991, Chapters 1.1 1.8.

(b) Hudlicky, M. Reductions in Organic Chemistry, 2nd ed., The American Chemical Society, Washington, DC, 1996.

[2] (a) Gunanathan, C.; Milstein, D. Science 2013, 341, 249.

(b) Ito, J.-I.; Nishiyama, H. Tetrahedron Lett. 2014, 55, 3133.

(c) Nandakumar, A.; Midya, S. P.; Landge, V. G.; Balaraman, E. Angew. Chem., Int. Ed. 2015, 54, 11022.

(d) Wang, D.; Astruc, D. Chem. Rev. 2015, 115, 6621.

(e) Ma, X.; Su, C.; Xu, Q. Top. Curr. Chem. 2016, 374, 27.

(f) Wang, C.; Xiao, J. Chem. Commun. 2017, 53, 3399.

(g) Corma, A.; Navas, J.; Sabater, M. J. Chem. Rev. 2018, 118, 1410 .

(h) Irrgang, T.; Kempe, R. Chem. Rev. 2019, 119, 2524.

[3] (a) Noyori, R.; Hashiguchi, S. Acc. Chem. Res. 1997, 30, 97. (b) Noyori, R.; Ohkuma, T. Angew. Chem., Int. Ed. 2001, 40, 40. (c) Noyori, R. Angew. Chem., Int. Ed. 2002, 41, 2008.

[4] (a) Baratta, W.; Ballico, M.; Chelucci, G.; Siega, K.; Rigo, P. Angew. Chem., Int. Ed. 2008, 47, 4362.

(b) Putignano, E.; Bossi, G.; Rigo, P.; Baratta, W. Organometallics 2012, 31, 1133.

(c) Chelucci, G.; Baldino, S.; Baratta, W. Acc. Chem. Res. 2015, 48, 363.

[5] (a) Morris, R. H. Chem. Soc. Rev. 2009, 38, 2282.

(b) Zuo, W.; Lough, A. J.; Li, Y. F.; Morris, R. H. Science 2013, $342,1080$.

(c) Morris, R. H. Acc. Chem. Res. 2015, 48, 1494.

(d) Seo, C. S. G.; Morris, R. H. Organometallics 2019, 38, 47.

[6] (a) Gunanathan, C.; Milstein, D. Chem. Rev. 2014, 114, 12024.

(b) Werkmeister, S.; Neumann, J.; Junge, K.; Beller, M. Chem.-Eur. J. 2015, 21, 12226.

(c) Kumar, A.; Bhatti, T. M.; Goldman, A. S. Chem. Rev. 2017, 117, 12357.

(d) Alig, L.; Fritz, M.; Schneider, S. Chem. Rev. 2019, 119, 2681.

[7] (a) Dani, P.; Karlen, T.; Gossage, R. A.; Gladiali, S.; van Koten, G. Angew. Chem., Int. Ed. 2000, 39, 743.

(b) Ito, J.-I.; Teshima, T.; Nishiyama, H. Chem. Commun. 2012, 48, 1105.

(c) Valdes, H.; Gonzalez-Sebastian, L.; Morales-Morales, D. J. Organomet. Chem. 2017, 845, 229.

[8] (a) Amoroso, D.; Jabri, A.; Yap, G. P. A.; Gusev, D. G.; dos Santos, E. N.; Fogg, D. E. Organometallics 2004, 23, 4047.

(b) Gagliardo, M.; Chase, P. A.; Brouwer, S.; Van Klink, G. P. M.; van Koten, G. Organometallics 2007, 26, 2219.

(c) Azerraf, C.; Gelman, D. Chem.-Eur. J. 2008, 14, 10364.

[9] Du, W.; Wang, L.; Wu, P.; Yu, Z. Chem.-Eur. J. 2012, 18, 11550.

[10] Zhang, G.; Hanson, S. K. Chem. Commun. 2013, 49, 10151.

[11] (a) He, L.-P.; Chen, T.; Xue, D.-X.; Eddaoudi, M.; Huang, K.-W. J. Organomet. Chem. 2012, 700, 202.

(b) Fuentes, J. A.; Carpenter, I.; Kann, N.; Clarke, M. L. Chem. Commun. 2013, 49, 10245.

[12] (a) Kannan, S.; Ramesh, P.; Liu, Y. J. Organomet. Chem. 2007, 692, 3380 .

(b) Maclnnis, M. C.; Maclean, D. F.; Lundgren, R. J.; McDonald, R.; Turculet, L. Organometallics 2007, 26, 6522.
[13] (a) Kannan, S.; Ramesh, P.; Liu, Y. J. Organomet. Chem. 2007, 692, 3380 .

(b) Maclnnis, M. C.; Maclean, D. F.; Lundgren, R. J.; McDonald, R.; Turculet, L. Organometallics 2007, 26, 6522.

(c) Qiu, Y.; Zhang, Y.; Jin, L.; Pan, L.; Du, G.; Ye, D.; Wang, D. Org. Chem. Front. 2019, 6, 3420 .

(d) Xu, Z.; Wang, D.; Yu, X.; Yang, Y.; Wang, D. Adv. Synth. Catal. 2017, 359, 3332 .

(e) Yao, W.; Duan, Z.; Zhang, Y.; Sang, X.; Xia, X.; Wang, D. Adv. Synth. Catal. 2019, 361, 5695.

(f) Hu, W.; Zhang, Y.; Zhu, H.; Ye, D.; Wang, D. Green Chem. 2019, 21, 5345 .

(g) Yang, Q.; Zhang, Y.; Zeng, W.; Duan, Z.; Sang, X.; Wang, D. Green Chem. 2019, 21, 5683.

(h) Yao, W.; Zhang, Y.; Zhu, H.; Wang, D. Chin. Chem. Lett. 2020, 31,701

(i) Tao, R.; Yang, Y.; Zhu, H.; Hu, X.; Wang, D. Green Chem. 2020, 22, DOI:10.1039/d0gc02341h.

(j) Wang, D.; Zhao, K.; Xu, C.; Miao, H.; Ding, Y. ACS Catal. 2014, 4, 3910.

(k) Ye, D.; Huang, R.; Zhu, H.; Zou, L.-H.; Wang, D. Org. Chem. Front. 2019, 6, 62

(1) Wu, Q.; Pan, L.; Du, G.; Zhang, C.; Wang, D. Org. Chem. Front. 2018, 5, 2668.

(m) Wang, D.; Yu, X.; Ge, B.; Miao, H.; Ding, Y. Chin. J. Org. Chem. 2015, 35, 676 (in Chinese).

(王大伟, 余晓丽, 葛冰洋, 苗红艳, 丁玉强, 有机化学, 2015, $35,676$.

[14] Cuervo, D.; Gamasa, M. P.; Gimeno, J. Chem.-Eur. J. 2004, 23, 425.

[15] Enthaler, S.; Hagemann, B.; Bhor, S.; Anilkumar, G.; Tse, M. K.; Bitterlich, B.; Junge, K.; Erre, G.; Beller, M. Adv. Synth. Catal. 2007, 349, 853 .

[16] (a) Moore, C. M.; Szymczak, N. K. Chem. Commun. 2013, 49, 400. (b) Shi, J.; Hu, B.; Gong, D.; Shang, S.; Hou, G.; Chen, D. Dalton Trans. 2016, 45, 4828.

[17] (a) Ye, W.; Zhao, M.; Du, W.; Jiang, Q.; Wu, K.; Wu, P.; Yu, Z. Chem.-Eur. J. 2011, 17, 4737.

(b) Ye, W.; Zhao, M.; Yu, Z. Chem.-Eur. J. 2012, 18, 10843.

(c) Jin, W.; Wang, L.; Yu, Z. Organometallics 2012, 31, 5664.

(d) Du, W.; Wu, P.; Wang, Q.; Yu, Z. Organometallics 2013, 32, 308.

(e) Du, W.; Wang, Q.; Wang, L.; Yu, Z. Organometallics 2014, 33, 974.

(f) Chai, H.; Liu, T.; Wang, Q.; Yu, Z. Organometallics 2015, 34, 5278 .

(g) Liu, T.; Chai, H.; Wang, L.; Yu, Z. Organometallics 2017, 36, 2914.

(h) Wang, Q.; Chai, H.; Yu, Z. Organometallics 2017, 36, 3638.

(i) Chai, H.; Liu, T.; Yu, Z. Organometallics 2017, 36, 4136.

(j) Chai, H.; Liu, T.; Zheng, D.; Yu, Z. Organometallics 2017, 36, 4268.

[18] (a) Yan, J.; Wang, Y.-B.; Zhu, Z.-H.; Li, Y.; Zhu, X.; Hao, X.-Q.; Song, M.-P. Organometallics 2018, 37, 2325.

(b) Wang, Y.-B.; Liu, Y.-X.; Zhu, Z.-H.; Zhao, X.-M.; Song, B.; Zhu, X.; Hao, X.-Q. J. Saudi Chem. Soc. 2019, 23, 104.

(c) Zhu, Z.-H.; Li, Y.; Wang, Y.-B.; Lan, Z.-G.; Zhu, X.; Hao, X.-Q.; Song, M.-P. Organometallics 2019, 38, 2156.

[19] (a) Li, K.; Niu, J.-L.; Yang, M.-Z.; Li, Z.; Wu, L.-Y.; Hao, X.-Q.; Song, M.-P. Organometallics 2015, 34, 1170.

(b) Yang, F.-L.; Zhu, X.; Rao, D.-K.; Cao, X.-N.; Li, K.; Xu, Y.; Hao, X.-Q.; Song, M.-P. RSC Adv. 2016, 6, 37093.

(c) Yang, F.-L.; Wang, Y.-H.; Ni, Y.-F.; Gao, X.; Song, B.; Zhu, X.; Hao, X.-Q. Eur. J. Org. Chem. 2017, 2017, 3481.

(d) Cao, X.-N.; Wan, X.-M.; Yang, F.-L.; Li, K.; Hao, X.-Q.; Shao, T.; Zhu, X.; Song, M.-P. J. Org. Chem. 2018, 83, 3657.

(e) Wan, X.-M.; Liu, Z.-L.; Liu, W.-Q.; Cao, X.-N.; Zhu, X.; Zhao, X.-M.; Song, B.; Hao, X.-Q.; Liu, G. Tetrahedron 2019, 75, 2697. 
[20] (a) Kröhnke, F.; Zecher, W.; Curtze, J.; Drechsler, D.; Pfleghar, K.; Schnalke, K. E.; Weis, C. W. Angew. Chem., Int. Ed. 1962, 1, 626.

(b) Ziegler, M.; Monney, V.; Stoeckli-Evans, H.; Zelewsky, A. V.; Sasaki, I.; Dupic, G.; Daran, J.-C.; Balavoine, G. G. A. J. Chem. Soc., Dalton Trans. 1999, 667

(c) Kwong, H.-L.; Lee, W.-S. Tetrahedron: Asymmetry 2000, 11, 2299.

(d) Kwong, H.-L.; Wong, W.-L.; Lee, W.-S.; Cheng, L.-S.; Wong, W.-T. Tetrahedron: Asymmetry 2001, 12, 2683.

(e) Yeung, C.-T.; Lee, W.-S.; Tsang, C.-S.; Yiu, S.-M.; Wong, W.-T.; Wong, W.-Y.; Kwong, H.-L. Polyhedron 2010, 29, 1497.

[21] (a) Wang, M.; Wang, C.; Hao, X.-Q.; Li, X.; Vaughn, T. J.; Zhang,
Y.-Y.; Yu, Y.; Li, Z.-Y.; Song, M.-P.; Yang, H.-B.; Li, X. J. Am. Chem. Soc. 2014, 136, 10499.

(b) Wang, L.; Liu, R.; Gu, J.; Song, B.; Wang, H.; Jiang, X.; Zhang, K.; Han, X.; Hao, X.-Q.; Bai, S.; Wang, M.; Li, X.; Xu, B.; Li, X. J. Am. Chem. Soc. 2018, 140, 14087.

[22] (a) Zhao, H.-Q.; Ong, W.-Q.; Fang, X.; Zhou, F.; Su, H.-B.; Zeng, H.-Q. Org. Biomol. Chem. 2012, 10, 1172.

(b) Ramogida, C. F.; Schindler, D.; Schneider, C.; Tan, Y. L. K.; Huh, S.; Ferreira, C. L.; Adam, M. J.; Orvig, C. RSC Adv. 2016, 6, 103763

(c) Gygi, D.; Hwang, S. J.; D.; Nocera, G. J. Org. Chem. 2017, 82, 12933.

(Zhao, C.) 